\title{
Surface Plasmon Enhanced Light Trapping in Metal/Silicon Nanobowl Arrays for Thin Film Photovoltaics
}

\author{
Ruinan Sun, Haoxin Fu, Jiang Wang, Yachun Wang, Xingchen Du, Haichuan Zhao, \\ Chenliang Huo, and Kuiqing Peng \\ Department of Physics and Beijing Key Laboratory of Energy Conversion and Storage Materials, Beijing Normal University, \\ Beijing 100875, China \\ Correspondence should be addressed to Kuiqing Peng; kq_peng@bnu.edu.cn
}

Received 10 March 2017; Revised 4 June 2017; Accepted 20 June 2017; Published 10 September 2017

Academic Editor: Jorge Pérez-Juste

Copyright (C) 2017 Ruinan Sun et al. This is an open access article distributed under the Creative Commons Attribution License, which permits unrestricted use, distribution, and reproduction in any medium, provided the original work is properly cited.

Enhancing the light absorption in thin film silicon solar cells with nanophotonic and plasmonic structures is important for the realization of high efficiency solar cells with significant cost reduction. In this work, we investigate periodic arrays of conformal metal/silicon nanobowl arrays (MSNBs) for light trapping applications in silicon solar cells. They exhibited excellent lightharvesting ability across a wide range of wavelengths up to infrared regimes. The optimized structure $\left(\mathrm{MSNBsH}^{2} \mathrm{covered}_{\mathrm{s}} \mathrm{SiO}_{2}\right.$ passivation layer and hemisphere $\mathrm{Ag}$ back reflection layer has a maximal short-circuit density $\left(J_{\mathrm{sc}}\right) 25.5 \mathrm{~mA} / \mathrm{cm}^{2}$, which is about $88.8 \%$ higher than flat structure counterpart, and the light-conversion efficiency $(\eta)$ is increased two times from $6.3 \%$ to $12.6 \%$. The double-side textures offer a promising approach to high efficiency ultrathin silicon solar cells.

\section{Introduction}

Solar energy is widely considered to be a reasonable solution for the loss of fossil fuels in the coming days. Silicon is always the leading material in commercial solar cells since it is the second most abundant material on earth and provides device reliability. Thin film solar cells have aroused significant interest due to the thickness reduction and low cost. The major disadvantage of thin film cells is the relatively poor absorption of light, particularly in the infrared part of the solar spectrum. Therefore, efficient light trapping structures are required in order to increase the light absorption length in the thin cell.

One approach proposed for achieving absorption enhancement is proper design of nanostructures such as nanocylinders [1], nanocones [2-4], nanodomes [5], nanosphere [6-9], nanobowl [10, 11], nanopillar [12], and hemispherical nanopit [13]. For example, silicon nanocylinder arrays were designed and fabricated; total reflectance spectroscopy showed an average reflectivity of only $1.3 \%$ for light in the wavelength of 450 900 $\mathrm{nm}$ range [1]. Wang et al. investigated the optical properties of $\mathrm{Si}$ nanocones arrays with various heights and diameters [4]. They showed that Si nanocones arrays can strongly interact with the incident light and the absorbance. In addition, silicon nanosphere on a high-index substrate shows narrow-band antireflection properties in the visible spectral range [6-8]. Gao et al. report a significant reflection reduction over a broadband light spectrum in crystalline silicon via introduction of low aspect-ratio honeycomb nanobowl front surface textures [11]. Yang et al. present the application of a conformal zig-zag nanostructure which shows significant absorption enhancement in the whole amorphous silicon [14].

Another approach proposed involves the excitation of localized surface plasmons in metal nanostructures [15] including metal nanogratings [16-18], metal nanodisk [19], and metal nanoparticles [20-24]. Ag and Au nanoparticles are the most widely used materials due to their surface plasmon resonances located in the visible range. The localized surface plasmon resonance (LSPR) of these metal nanoparticles results in strong light trapping and strongly enhanced near-field electric field distributions around the particle [2530]. In previously reported arrays of silicon nanobowl arrays (SNBs) embedded with metal nanoparticles [31], the overall absorption loss is high due to serious light-decay in metal nanoparticles, leading to low power conversion efficiency. 
This absorption loss problem has been well addressed by changing metal nanoparticles to hemisphere metal nanobowl shell. We found that a thin metal shell on top of the silicon nanobowl arrays also has effective light trapping ability. The excitation of surface plasmon resonances in metal nanobowl shell can increase the energy transmitted into the silicon layer at the same time. With the huge decrease of metal mass, serious light-decay in metal nanoparticles which cause high light absorption is fixed in MSNBs.

In this paper, we demonstrate that broadband solar absorption enhancement across a wide range of wavelengths up to infrared regimes can be achieved by employing a periodic arrays of conformal metal/silicon nanobowl arrays (MSNBs) design. Such nanophotonic-plasmonic structure can be produced using well-established metal-assisted electroless etching technique [32-34]. We study the effect of bowl size, metal choice, and hemisphere Ag back reflection for optimizing the light trapping efficiency and solar cell performance.

\section{Simulation Methods}

In this work, the finite-difference time-domain (FDTD) method was applied to calculate the absorption per unit volume in silicon layer from the divergence of the Poynting vector $\vec{P}$,

$$
\begin{aligned}
& P_{\mathrm{abs}}=-0.5 \operatorname{real}(\vec{\nabla} \cdot \vec{P}), \\
& P_{\mathrm{abs}}=-0.5 \omega|E|^{2} \operatorname{imag}(\varepsilon) .
\end{aligned}
$$

To calculate the absorption as a function of space and frequency, we only need to know the electric field intensity $(E)$ and the imaginary part of the permittivity $(\varepsilon)$. Both quantities are easy to measure in an FDTD simulation. The number of absorbed photons per unit volume $(g)$ can be calculated by dividing this value by the energy per photon,

$$
g=\frac{P_{\mathrm{abs}}}{\hbar \omega}=\frac{-0.5|E|^{2} \operatorname{imag}(\varepsilon)}{\hbar} .
$$

The short-circuit current density $J_{\mathrm{sc}}$ can directly be calculated from the formula

$$
J_{\mathrm{sc}}=q \int E Q E(\lambda) S(\lambda) d \lambda=q \int \frac{P_{\mathrm{abs}}(\lambda)}{P_{\mathrm{in}}(\lambda)} S(\lambda) d \lambda,
$$

where $q$ is the electron charge and $S(\lambda)$ is the standard spectral photon density of sunlight at the earth's surface air mass of 1.5.

To evaluate the performance of the solar cell, we typically measure the photovoltaic power conversion efficiency,

$$
\eta=\frac{\mathrm{FF} \times V_{\mathrm{oc}} \times J_{\mathrm{sc}}}{S_{\mathrm{AM} 1.5 \mathrm{G}}}=\frac{P_{\max }}{S_{\mathrm{AM} 1.5 \mathrm{G}}} .
$$

where $S_{\mathrm{AM} 1.5 \mathrm{G}}$ is the incident power from the AM1.5G solar model: $100 \mathrm{~mW} / \mathrm{cm}^{2}$. $J_{\mathrm{sc}}$ is short-circuit density, $V_{\mathrm{oc}}$ is open circuit voltage, and FF is fill factor of the solar cell.

\section{Results and Discussion}

Figure 1(a) illustrates the diagram of the metal/silicon nanobowl arrays (MSNBs); Figure 1(b) is the cross-sectional view of MSNBs. The thickness of silicon layer is predefined $L=400 \mathrm{~nm}$; two parameters, the diameter of metal bowl $D$ and thickness of metal bowl shell $d$, are left to control the light trapping performance. Figure 1(c) is optimized structure of MSNBs covered by $\mathrm{SiO}_{2}$ passivation layer and hemisphere $\mathrm{Ag}$ back reflection layer which we named MSNBsH. The crosssectional view of new structure is shown in Figure $1(d)$. The thickness of silicon layer in $\mathrm{MSNBsH}$ is predefined $L=$ $1000 \mathrm{~nm}, \mathrm{SiO}_{2}$ passivation layer $d_{1}=d_{2}=10 \mathrm{~nm}$, and thickness of metal bowl shell is $d_{A}$. The sunlight causes illumination from the top surface of MSNBs along $Z$ direction. The boundary conditions used in the simulation area are perfectly matched layers (PMLs) in $x y$ plane and periodic boundary conditions (PBCs) in $x z$ and $y z$ plane. The minimum mesh space is $1 \mathrm{~nm}$ near the metal where the electric field changes rapidly. In this way, the simulation results will be more accurate. A nonabsorbing $\mathrm{SiO}_{2}$ layer was used with the real part of its refractive index data. The refractive index data and absorption coefficient of $\mathrm{Si}, \mathrm{SiO}_{2}, \mathrm{Au}$, and $\mathrm{Ag}$ were obtained from Palik [35].

Figure 2(a) shows the calculated short-current density $J_{\text {sc }}$ of MSNBs, SNBs, and flat silicon structure as a function of thickness of metal bowl shell $d$. It is observed that larger diameter Au bowl shell $(D=300 \mathrm{~nm})$ with $5 \mathrm{~nm}$ thickness has maximum short-current density $J_{\mathrm{sc}}=23.7 \mathrm{~mA} / \mathrm{cm}^{2}$, compared to nanobowl array whose maximum $J_{\mathrm{sc}}=$ $15.7 \mathrm{~mA} / \mathrm{cm}^{2}$ has an enhancement of $50.9 \%$. The simulation results reveal that the structure with larger diameter has higher light absorption efficiency. We found that, under the $400 \mathrm{~nm}$ thick silicon layer, the transmission light is still high which will cause solar energy loss in MSNBs structure. Optimized structure of MSNBs covered by $\mathrm{SiO}_{2}$ passivation layer and hemisphere Ag back reflection layer will fix the problem. Figure 2(b) exhibits short-current density $J_{\mathrm{sc}}$ of MSNBsH as a function of diameter of metal bowl $D$. Also SNBs covered by $\mathrm{SiO}_{2}$ passivation layer and hemisphere Ag back reflection layer and flat silicon structure covered by $\mathrm{SiO}_{2}$ passivation layer and hemisphere $\mathrm{Ag}$ back reflection layer are plotted for comparison. It is shown that $J_{\mathrm{sc}}$ first increase and then decrease with increasing diameter metal bowl shell $D$. When $D=300 \mathrm{~nm}$, Au bowl shell with $5 \mathrm{~nm}$ thickness has highest short-current density $J_{\mathrm{sc}}=28.5 \mathrm{~mA} / \mathrm{cm}^{2}$. The result indicates that in both MSNBs and MSNBsH structure $\mathrm{Au}$ bowl shell has slightly higher short-current density than Ag bowl shell. As we all know, the large refractive index difference could result in high reflection when light moves between media of different refractive indices. The effective refractive index of Au nanobowl arrays changes gradually compared to Ag nanobowl arrays owing to Au having higher refractive index than Ag in the wavelength of visible light.

Figure 3(a) shows absorption spectra in silicon layer of the MSNBsH with thickness of metal bowl shell $d_{A}=5 \mathrm{~nm}$. It can be clearly observed that MSNBsH shows dramatically enhanced absorption over whole solar spectra especially in infrared range. The absorption result indicates that $\mathrm{Au}$ 


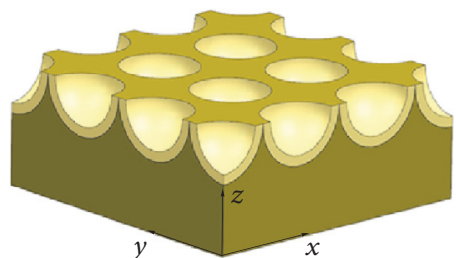

$\mathrm{Au}$

$\mathrm{Si}$

(a)

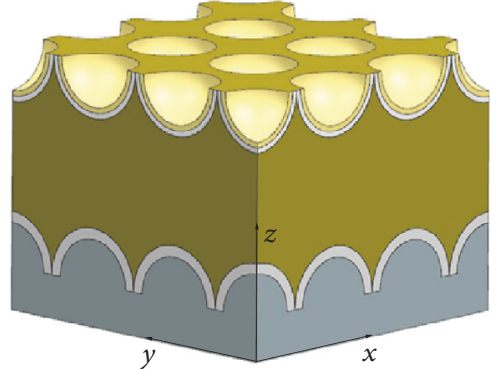

$\mathrm{Au}$

$\mathrm{Si}$
$\mathrm{SiO}_{2}$

(c)

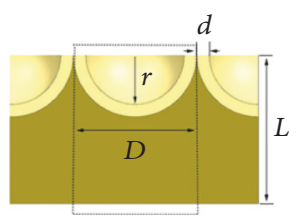

$\mathrm{Au}$

$\mathrm{Si}$

(b)

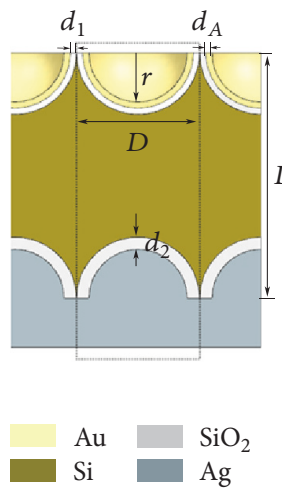

(d)

FIGURE 1: (a) Schematic illustration of metal/silicon nanobowl arrays (MSNBs); (b) two-dimensional side view of MSNBs; (c) MSNBs covered by $\mathrm{SiO}_{2}$ passivation layer and hemisphere Ag back reflection layer (MSNBsH); (d) cross-sectional view of MSNBsH. Light is incident on the top of the structure along the $z$ direction.

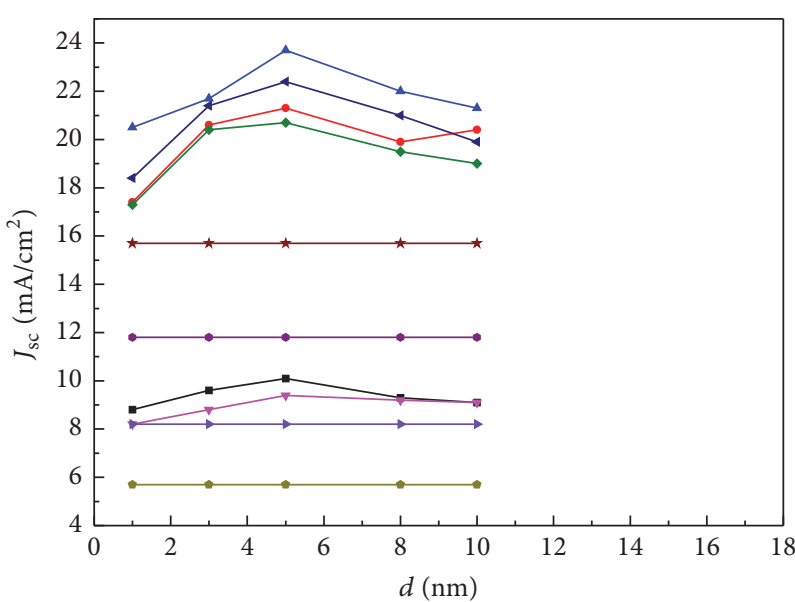

$\longrightarrow$ Au/SNBs, $D=100 \mathrm{~nm} \quad \longrightarrow$ Ag/SNBs, $D=300 \mathrm{~nm}$
$\longrightarrow$ Au/SNBs, $D=200 \mathrm{~nm} \quad \longrightarrow$ SNBs, $D=100 \mathrm{~nm}$
$\longrightarrow$ Au/SNBs, $D=300 \mathrm{~nm} \rightarrow$ SNBs, $D=200 \mathrm{~nm}$
$\longrightarrow$ Ag/SNBs, $D=100 \mathrm{~nm} \rightarrow$ SNBs, $D=300 \mathrm{~nm}$
$\longrightarrow$ Ag/SNBs, $D=200 \mathrm{~nm} \quad \longrightarrow$ Flat

(a)

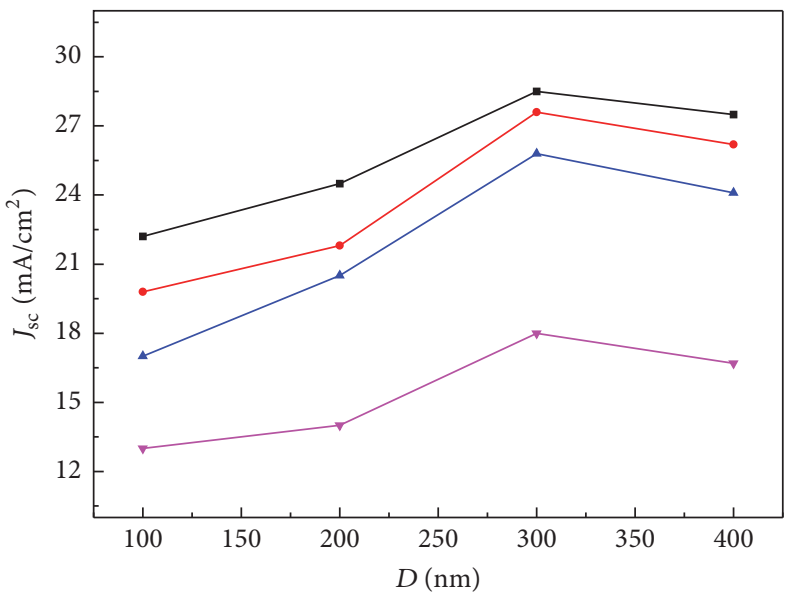

$\rightarrow \mathrm{Au} / \mathrm{SNBsH}, d_{A}=5 \mathrm{~nm}$
$\rightarrow \mathrm{Ag} / \mathrm{SNBsH}, d_{A}=5 \mathrm{~nm}$
$\simeq \mathrm{SNBsH}$
$\rightarrow$ Flat

(b)

FIGURE 2: (a) Calculated short-current density $J_{\mathrm{sc}}$ of MSNBs, SNBs, and flat silicon structure as a function of thickness of metal bowl shell $d$. (b) Calculated short-current density $J_{\text {sc }}$ of $\mathrm{MSNBsH}, \mathrm{SNBs}$ with $\mathrm{SiO}_{2}$ passivation layer and hemisphere Ag back reflector ( $\mathrm{SNBsH}$ ), and flat structure covered by $\mathrm{SiO}_{2}$ passivation layer and hemisphere Ag back reflection layer as a function of diameter of metal bowl $D$. 

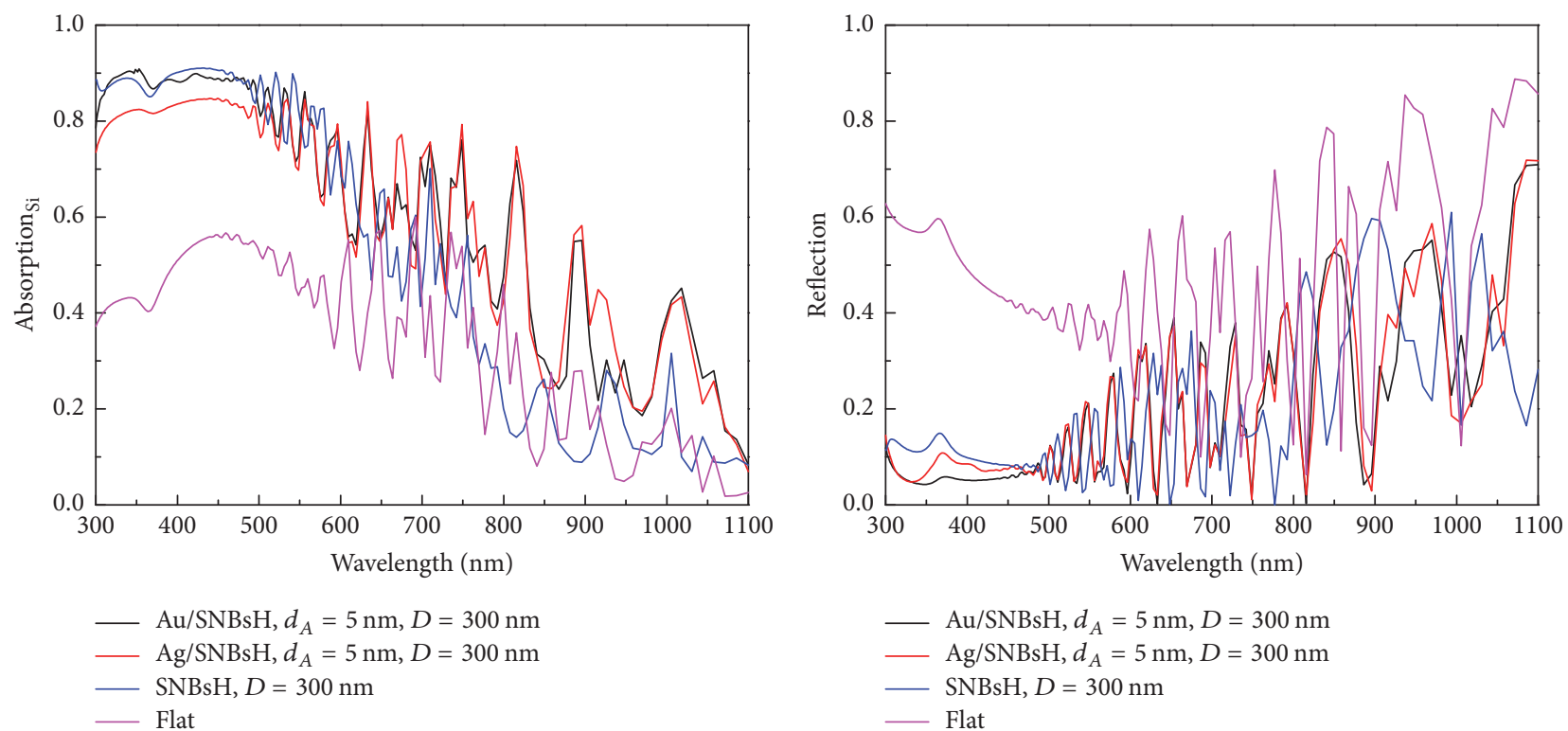

(a)

(b)

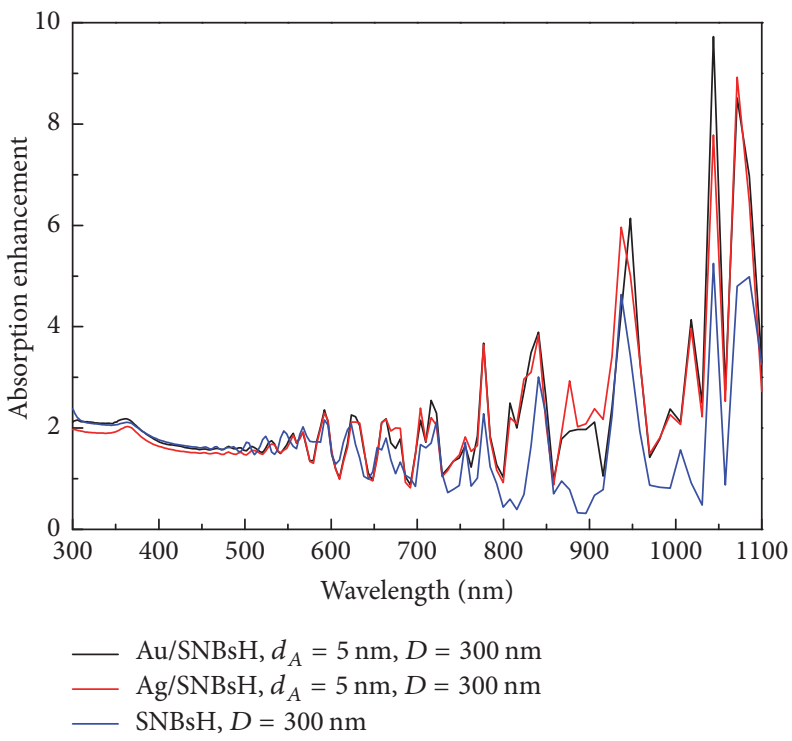

(c)

Figure 3: (a) Wavelength dependence of absorption spectra in silicon layer of the MSNBsH with thickness of metal bowl shell $d_{A}=5 \mathrm{~nm}$ and diameter $D=300 \mathrm{~nm}$. (b) Reflection spectra; (c) absorption enhancement spectra generated by normalizing the absorption of MSNBsH to the absorption of flat silicon structure.

bowl shell is slightly better than Ag bowl shell when the wavelength $\lambda<500 \mathrm{~nm}$. The flat silicon structure covered by $\mathrm{SiO}_{2}$ passivation layer and hemisphere $\mathrm{Ag}$ back reflect reflection is also included for comparison which has much lower absorption in whole wavelength. The reflection spectra of MSNBsH are plotted in Figure 3(b). The MSNBsH array exhibits excellent light trapping property across the whole wavelength range investigated and higher solar absorption than silicon nanobowl arrays over the wavelength range from 700 to $1100 \mathrm{~nm}$. The overall absorption efficiency of optimized MSNBsH array is higher than that of the flat silicon structure, implying a stronger coupling between the incident light and modes supported in the MSNBsH array structure. The absorption enhancement in silicon layer of $\mathrm{MSNBsH}$ arrays compared with the flat silicon structure is shown in Figure 3(c). The enhancement rate is almost smooth when $\lambda<500 \mathrm{~nm}$, due to the excellent light trapping property and low light reflection effect of MSNBsH, while in infrared wavelength multiple sharp peaks appear as various kinds of optical resonances.

In order to understand the full optical performance of the MSNBsH array, the carrier generation rates in silicon layer weighted by solar spectrum calculated by the FDTD simulations are shown in Figures $4(\mathrm{a})-4(\mathrm{~d})$. For $5 \mathrm{~nm}$ thick 


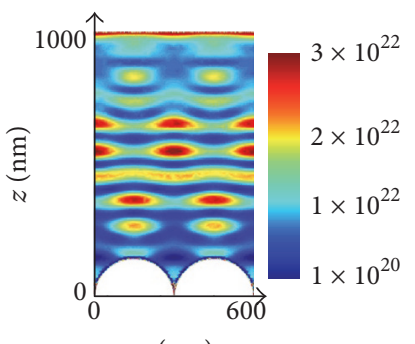

(a)

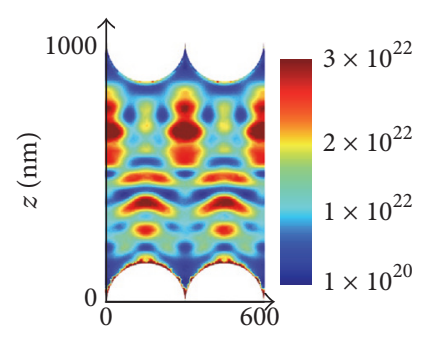

$x(\mathrm{~nm})$

(b)

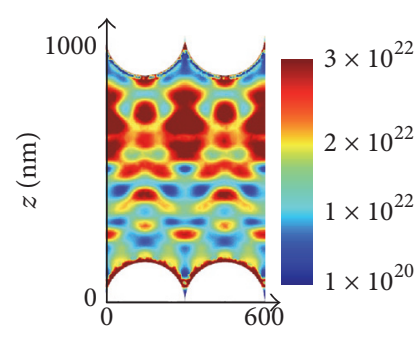

$x(\mathrm{~nm})$

(c)

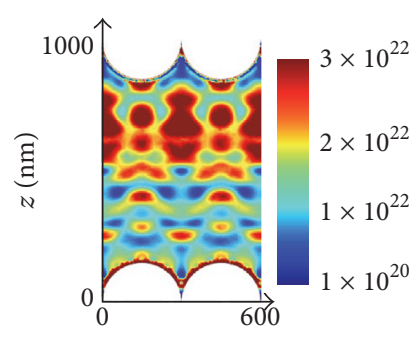

$x(\mathrm{~nm})$

(d)

FIGURE 4: Calculated generation rate $\left(\mathrm{cm}^{-3} \mathrm{~s}^{-1}\right)$ in silicon weighted by solar spectrum. (a) Flat silicon structure with $\mathrm{SiO}_{2}$ passivation layer and hemisphere $\mathrm{Ag}$ back reflector, (b) silicon nanobowl arrays with $\mathrm{SiO}_{2}$ passivation layer and hemisphere Ag back reflector, (c) MSNBsH with Au bowl shell $d_{A}=5 \mathrm{~nm}$, and (d) MSNBsH with Ag bowl shell $d_{A}=5 \mathrm{~nm}$.

TABLE 1: Device performance of different solar cells via actual device simulations.

\begin{tabular}{|c|c|c|c|c|c|}
\hline & Nanobowl diameter $(\mathrm{nm})$ & $V_{\text {oc }}(\mathrm{v})$ & $J_{\mathrm{sc}}\left(\mathrm{mA} / \mathrm{cm}^{2}\right)$ & $\mathrm{FF}(\%)$ & $\eta(\%)$ \\
\hline Flat & & 0.56 & 13.5 & 83.3 & 6.3 \\
\hline SNBsH & 300 & 0.58 & 22.8 & 83.2 & 11.0 \\
\hline $\mathrm{Au} / \mathrm{SNBsH}$ & 300 & 0.59 & 25.5 & 83.8 & 12.6 \\
\hline $\mathrm{Ag} / \mathrm{SNBsH}$ & 300 & 0.58 & 24.9 & 83.7 & 12.1 \\
\hline
\end{tabular}

$\mathrm{Au} / \mathrm{Ag}$ bowl shell with diameter $\mathrm{D}=300 \mathrm{~nm}$, high carrier generation rates in almost whole photoactive silicon layer are observed in Figures 4(c) and 4(d). Comparing with silicon nanobowl arrays with $\mathrm{SiO}_{2}$ passivation layer and hemisphere $\mathrm{Ag}$ back reflector in Figure 4(b), the excitation of surface plasmon resonances in metal nanobowl shell can increase the energy transmitted into the silicon layer. The localized surface plasmon resonance (LSPR) of these metal hemisphere shells results in strong light trapping and a strongly enhanced near-field electric field distribution in the $\mathrm{Au} / \mathrm{Ag}$ shell. What is more, the infrared incident sunlight was reflected between metal nanobowl shell and metal hemisphere Ag back reflection layer, redirecting light absorption into the cell and potentially coupling into waveguide modes of the device. The unique light trapping geometries described here can couple light preferentially into the silicon film. Therefore the path length of the incident light and the waveguide modes in MSNBsH structure are both increased [36].

In optical design, we assume that the internal quantum efficiency IQE = $100 \%$, while there are several kinds of carrier losses such as Auger and bimolecular radiative recombinations that should be considered on the design of thin film solar cells. To understand the effect of enhanced solar absorption on photovoltaic devices, we conduct solar cells optoelectronic simulation [37]. Here, the carrier transport process is based on the carrier generation rates calculated from optical simulation shown in Figure 4. The electrical parameters of MSNBsH solar cells designed are as follows: a $1000 \mathrm{~nm}$ thick silicon as the active layer, a $200 \mathrm{~nm}$ thick $\mathrm{Au}$ grid as the front transparent conductive electrode, and a $200 \mathrm{~nm}$ thick Ag grid as the back electrode. The $n$ and $p$ doping concentration are both $1 \times 10^{19} \mathrm{~cm}^{-3}$. The location of $n$ region is along $Z$ direction $[800,1000] \mathrm{nm}, i$ region $[200,800] \mathrm{nm}$, and $p$ region $[0,200] \mathrm{nm}$. The thicknesses of $n$ and $p$ regions are both $200 \mathrm{~nm}$. The coefficients of Auger and bimolecular radiative recombinations are hole $9.9 \times 10^{-32} \mathrm{~cm}^{6} / \mathrm{s}$ and electron $2.8 \times 10^{-31} \mathrm{~cm}^{6} / \mathrm{s}$ and $1.64 \times$ $10^{-14} \mathrm{~cm}^{3} / \mathrm{s}$ (see Figure 5(a)).

In this device simulation we calculated the short-circuit density $\left(J_{\mathrm{sc}}\right)$, open circuit voltage $\left(V_{\mathrm{oc}}\right)$, fill factor $(\mathrm{FF})$, and power conversion efficiency of flat silicon arrays covered by $\mathrm{SiO}_{2}$ passivation layer and hemisphere Ag back reflection layer and $\mathrm{SiNB}$ covered by $\mathrm{SiO}_{2}$ passivation layer and hemisphere $\mathrm{Ag}$ back reflection layer ( $\mathrm{SNBsH}$ ) and $\mathrm{MSNBsH}$ structures (Table 1). All the diameter of hemisphere Ag back reflection layer $D=300 \mathrm{~nm}$. Figure 5(b) shows the current-voltage $(I-V)$ simulation results of different optimized structure-based devices under AM1.5G solar spectrum illumination. Compared to flat silicon structure, $5 \mathrm{~nm}$ thick Au bowl shell with diameter $D=300 \mathrm{~nm}$ exhibits an $88.8 \%$ increase in short-circuit density $\left(J_{\mathrm{sc}}\right)$ from $13.5 \mathrm{~mA} / \mathrm{cm}^{2}$ to $25.5 \mathrm{~mA} / \mathrm{cm}^{2}, 5 \%$ increase of open circuit voltage $\left(V_{\mathrm{oc}}\right)$, and significantly increased light-conversion efficiency $(\eta)$ from $6.3 \%$ to $12.6 \%$ in Figure 5(c).

\section{Conclusions}

In summary, we have proposed periodic arrays of conformal metal/silicon nanobowl arrays (MSNBs) and MSNBs covered by $\mathrm{SiO}_{2}$ passivation layer and hemisphere Ag back reflection layer (MSNBsH) for light trapping applications in thin silicon solar cells. Such metal/silicon nanobowl arrays exhibited excellent light-harvesting capability across a wide range of wavelengths up to infrared regimes. The analysis reveals that the efficient solar absorption is due to the effective optical coupling between the silicon nanobowl arrays and the incident sunlight and enhanced optical resonances between metal nanobowl shell and the back hemisphere Ag back 

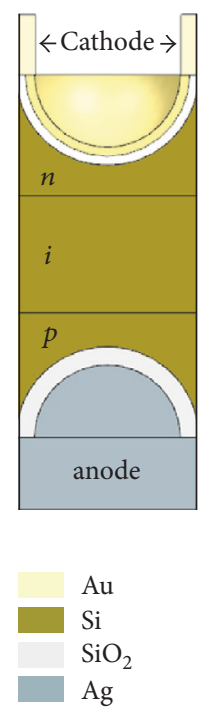

(a)

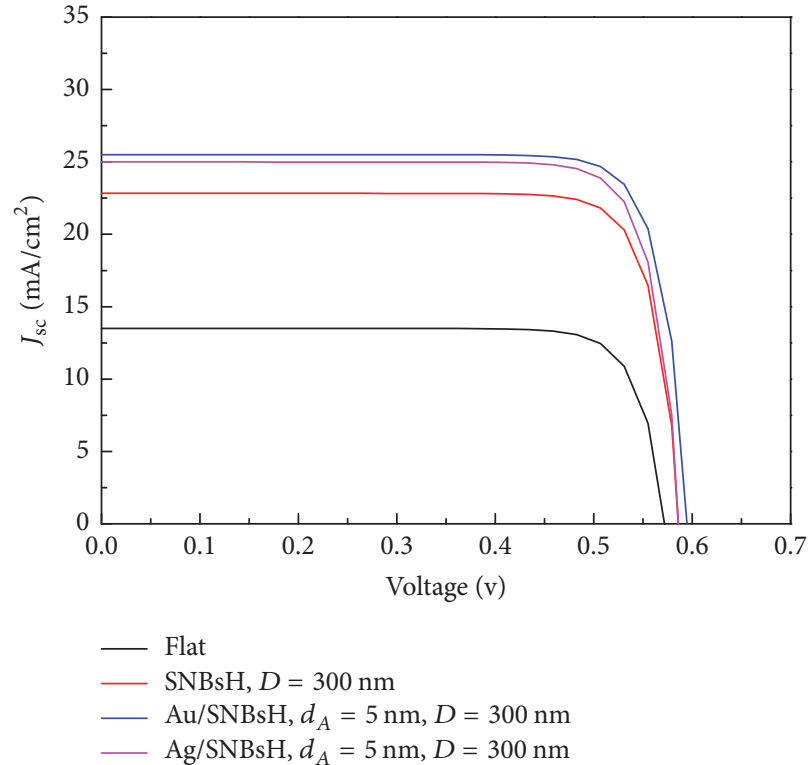

(b)

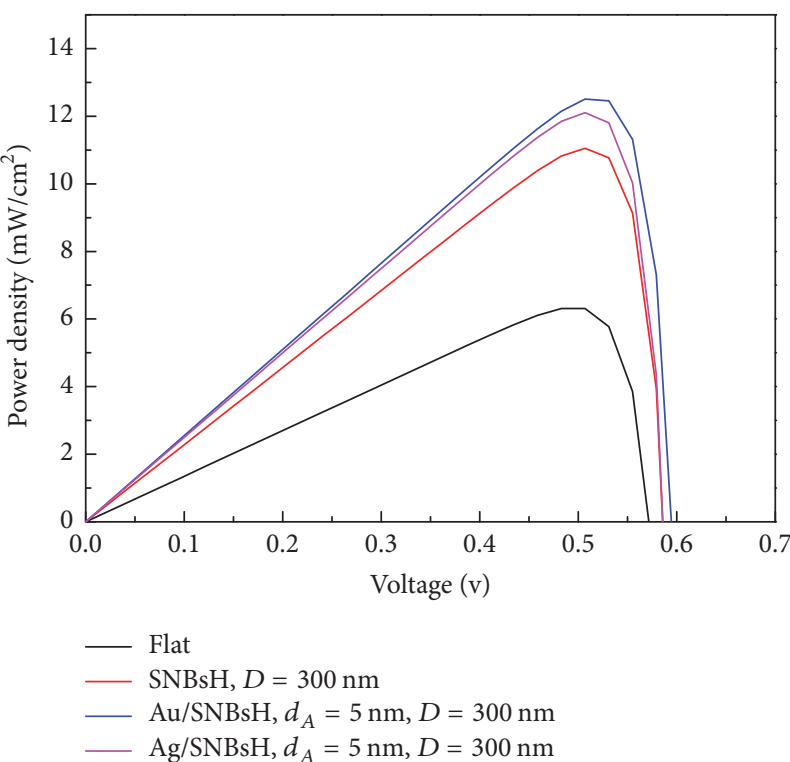

(c)

Figure 5: (a) Schematic illustration of MSNBsH in electrical simulation, (b) the current-voltage ( $I-V)$ simulation results, and (c) power density results of different structure-based devices under the illumination of AM1.5G solar spectrum. The blue curve is the typical $I-V$ curve for structure with $\mathrm{Au} / \mathrm{SNBsH}$ structure which has highest short-circuit density and power conversion efficiency. The pink curve is for structure with $\mathrm{Ag} / \mathrm{SNBsH}$ structure which has highest short-circuit density and power conversion efficiency. The red curve is the SNBs structure with hemisphere Ag back reflector and the black one is the $1000 \mathrm{~nm}$ flat silicon structure with hemisphere Ag back reflector for comparison.

reflection layer, especially the electric field intensity enhancement around metal bowl shell due to the localized surface plasmon resonance (LSPR). The optimized structure yields a short-circuit density $\left(J_{\mathrm{sc}}\right)$ up to $25.5 \mathrm{~mA} / \mathrm{cm}^{2}$, which is about $88.8 \%$ higher than flat silicon structure counterpart, and the light-conversion efficiency $(\eta)$ is increased two times from $6.3 \%$ to $12.6 \%$. We found that both $\mathrm{Au}$ and Ag lead to really similar light trapping ability, but Au has a better performance in both optical and electrical stimulation. We suggest that such metal/silicon nanobowl array design provides a promising strategy in conversion efficiency improvement and cost reduction for thin silicon solar cells.

\section{Conflicts of Interest}

The authors declare that there are no conflicts of interest regarding the publication of this paper.

\section{Acknowledgments}

This work was supported by the National Natural Science Foundation of China (91333208), Beijing Natural Science 
Foundation (2172030), NSFC (51072025), and the Fundamental Research Funds of the Central Universities (2012LZD02).

\section{References}

[1] P. Spinelli, M. A. Verschuuren, and A. Polman, "Broadband omnidirectional antireflection coating based on subwavelength surface Mie resonators," Nature Communications, vol. 3, article no. $692,2012$.

[2] S. Jeong, M. D. McGehee, and Y. Cui, "All-back-contact ultrathin silicon nanocone solar cells with $13.7 \%$ power conversion efficiency," Nature Communications, vol. 4, article 2950, 2013.

[3] K. X. Wang, Z. Yu, V. Liu, Y. Cui, and S. Fan, "Absorption enhancement in ultrathin crystalline silicon solar cells with antireflection and light-trapping nanocone gratings," Nano Letters, vol. 12, no. 3, pp. 1616-1619, 2012.

[4] Z. Y. Wang, R. J. Zhang, S. Y. Wang et al., "Broadband optical absorption by tunable Mie resonances in silicon nanocone arrays," Scientific Reports, vol. 5, article no. 7810, 2015.

[5] J. Zhu, C.-M. Hsu, Z. Yu, S. Fan, and Y. Cui, "Nanodome solar cells with efficient light management and self-cleaning," Nano Letters, vol. 10, no. 6, pp. 1979-1984, 2010.

[6] K. V. Baryshnikova, M. I. Petrov, V. E. Babicheva, and P. A. Belov, "Plasmonic and silicon spherical nanoparticle antireflective coatings," Scientific Reports, vol. 6, Article ID 22136, 2016.

[7] H. Lin, F. Xiu, M. Fang et al., "Rational design of inverted nanopencil arrays for cost-effective, broadband, and omnidirectional light harvesting," ACS Nano, vol. 8, no. 4, pp. 3752-3760, 2014.

[8] Z. Yang, P. Gao, C. Zhang, X. Li, and J. Ye, "Scattering effect of the high-index dielectric nanospheres for high performance hydrogenated amorphous silicon thin-film solar cells," Scientific Reports, vol. 6, Article ID 30503, 2016.

[9] P. Gao, J. He, S. Zhou et al., "Large-Area Nanosphere SelfAssembly by a Micro-Propulsive Injection Method for High Throughput Periodic Surface Nanotexturing," Nano Letters, vol. 15, no. 7, pp. 4591-4598, 2015.

[10] Z. Yang, X. Li, S. Wu, P. Gao, and J. Ye, "High-efficiency photon capturing in ultrathin silicon solar cells with front nanobowl texture and truncated-nanopyramid reflector," Optics Letters, vol. 40, no. 6, pp. 1077-1080, 2015.

[11] P. Gao, H. Wang, Z. Sun, W. Han, J. Li, and J. Ye, "Efficient light trapping in low aspect-ratio honeycomb nanobowl surface texturing for crystalline silicon solar cell applications," Applied Physics Letters, vol. 103, no. 25, Article ID 253105, 2013.

[12] J. Li, H. Yu, S. Wong et al., "Si nanopillar array optimization on Si thin films for solar energy harvesting," Applied Physics Letters, vol. 95, Article ID 033102, 2009.

[13] J. Li, H. Yu, Y. Li, F. Wang, M. Yang, and S. M. Wong, "Low aspect-ratio hemispherical nanopit surface texturing for enhancing light absorption in crystalline Si thin film-based solar cells," Applied Physics Letters, vol. 98, no. 2, Article ID 021905, 2011.

[14] Z. Yang, A. Shang, Y. Zhan, C. Zhang, and X. Li, "Ultrabroadband performance enhancement of thin-film amorphous silicon solar cells with conformal zig-zag configuration," Optics Letters, vol. 38, no. 23, pp. 5071-5074, 2013.

[15] H. A. Atwater and A. Polman, "Plasmonics for improved photovoltaic devices," Nature Materials, vol. 9, no. 3, pp. 205$213,2010$.
[16] A. Abass, K. Q. Le, A. Alù, M. Burgelman, and B. Maes, "Dualinterface gratings for broadband absorption enhancement in thin-film solar cells," Physical Review B - Condensed Matter and Materials Physics, vol. 85, no. 11, Article ID 115449, 2012.

[17] L. Wen, F. Sun, and Q. Chen, "Cascading metallic gratings for broadband absorption enhancement in ultrathin plasmonic solar cells," Applied Physics Letters, vol. 104, no. 15, Article ID 151106, 2014.

[18] R. A. Pala, J. White, E. Barnard, J. Liu, and M. L. Brongersma, "Design of plasmonic thin-film solar cells with broadband absorption enhancements," Advanced Materials, vol. 21, no. 34, pp. 3504-3509, 2009.

[19] C. Hägglund, M. Zäch, G. Petersson, and B. Kasemo, "Electromagnetic coupling of light into a silicon solar cell by nanodisk plasmons," Applied Physics Letters, vol. 92, no. 5, Article ID 053110, 2008

[20] F. J. Beck, S. Mokkapati, A. Polman, and K. R. Catchpole, "Asymmetry in photocurrent enhancement by plasmonic nanoparticle arrays located on the front or on the rear of solar cells," Applied Physics Letters, vol. 96, no. 3, Article ID 033113, 2010.

[21] D. Derkacs, S. H. Lim, P. Matheu, W. Mar, and E. T. Yu, "Improved performance of amorphous silicon solar cells via scattering from surface plasmon polaritons in nearby metallic nanoparticles," Applied Physics Letters, vol. 89, Article ID 093103, 2006.

[22] J. Park, N. Park, and S. Varlamov, “Optimum surface condition for plasmonic Ag nanoparticles in polycrystalline silicon thin film solar cells," Applied Physics Letters, vol. 104, no. 3, Article ID 033903, 2014.

[23] P. Spinelli and A. Polman, "Prospects of near-field plasmonic absorption enhancement in semiconductor materials using embedded Ag nanoparticles," Optics Express, vol. 20, no. 105, pp. A641-A654, 2012.

[24] H. Tan, R. Santbergen, A. H. M. Smets, and M. Zeman, "Plasmonic light trapping in thin-film silicon solar cells with improved self-assembled silver nanoparticles," Nano Letters, vol. 12, no. 8, pp. 4070-4076, 2012.

[25] J. Bhattacharya, N. Chakravarty, S. Pattnaik, W. Dennis Slafer, R. Biswas, and V. L. Dalal, "A photonic-plasmonic structure for enhancing light absorption in thin film solar cells," Applied Physics Letters, vol. 99, no. 13, Article ID 131114, 2011.

[26] A. Basch, F. J. Beck, T. Söderström, S. Varlamov, and K. R. Catchpole, "Combined plasmonic and dielectric rear reflectors for enhanced photocurrent in solar cells," Applied Physics Letters, vol. 100, no. 24, Article ID 243903, 2012.

[27] M. Van Lare, F. Lenzmann, and A. Polman, "Dielectric back scattering patterns for light trapping in thin-film Si solar cells," Optics Express, vol. 21, no. 18, pp. 20738-20746, 2013.

[28] Z. Ouyang, S. Pillai, F. Beck et al., "Effective light trapping in polycrystalline silicon thin-film solar cells by means of rear localized surface plasmons," Applied Physics Letters, vol. 96, no. 26, Article ID 261109, 2010.

[29] M. Yang, Z. Fu, F. Lin, and X. Zhu, "Incident angle dependence of absorption enhancement in plasmonic solar cells," Optics Express, vol. 19, no. 104, pp. A763-A771, 2011.

[30] M. Van Lare, F. Lenzmann, M. A. Verschuuren, and A. Polman, "Mode coupling by plasmonic surface scatterers in thin-film silicon solar cells," Applied Physics Letters, vol. 101, no. 22, Article ID 221110, 2012.

[31] R.-N. Sun, K.-Q. Peng, B. Hu, Y. Hu, F.-Q. Zhang, and S.-T. Lee, "Plasmon enhanced broadband optical absorption in ultrathin 
silicon nanobowl array for photoactive devices applications," Applied Physics Letters, vol. 107, no. 1, Article ID 013107, 2015.

[32] X. Li and P. W. Bonn, "Metal-assisted chemical etching in $\mathrm{HF} / \mathrm{H}_{2} \mathrm{O}_{2}$ produces porous silicon," Applied Physics Letters, vol. 77, no. 16, pp. 2572-2574, 2000.

[33] K. Peng, A. Lu, R. Zhang, and S. T. Lee, "Motility of metal nanoparticles in silicon and induced anisotropic silicon etching," Advanced Functional Materials, vol. 18, no. 19, pp. 30263035, 2008.

[34] K. Peng, X. Wang, X. Wu, and S. Lee, "Fabrication and photovoltaic property of ordered macroporous silicon," Applied Physics Letters, vol. 95, no. 14, p. 143119, 2009.

[35] E. D. Palik, Handbook of Optical Constants of Solids, Elsevier, London, UK, 1988.

[36] M. G. Deceglie, V. E. Ferry, A. P. Alivisatos, and H. A. Atwater, "Design of nanostructured solar cells using coupled optical and electrical modeling," Nano Letters, vol. 12, no. 6, pp. 2894-2900, 2012.

[37] X. Li and Y. Zhan, "Enhanced external quantum efficiency in rectangular single nanowire solar cells," Applied Physics Letters, vol. 102, no. 2, Article ID 021101, 2013. 

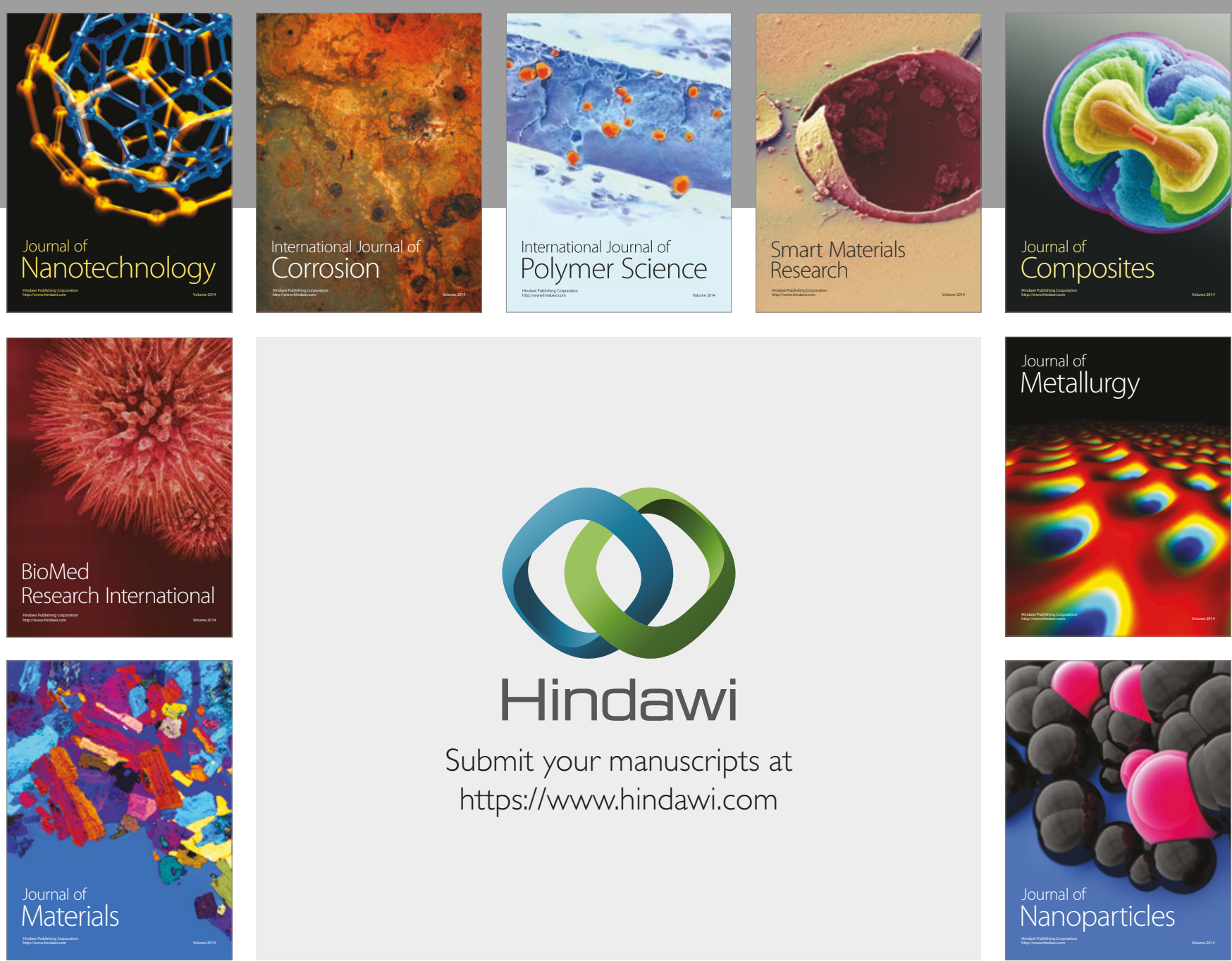

\section{Hindawi}

Submit your manuscripts at

https://www.hindawi.com
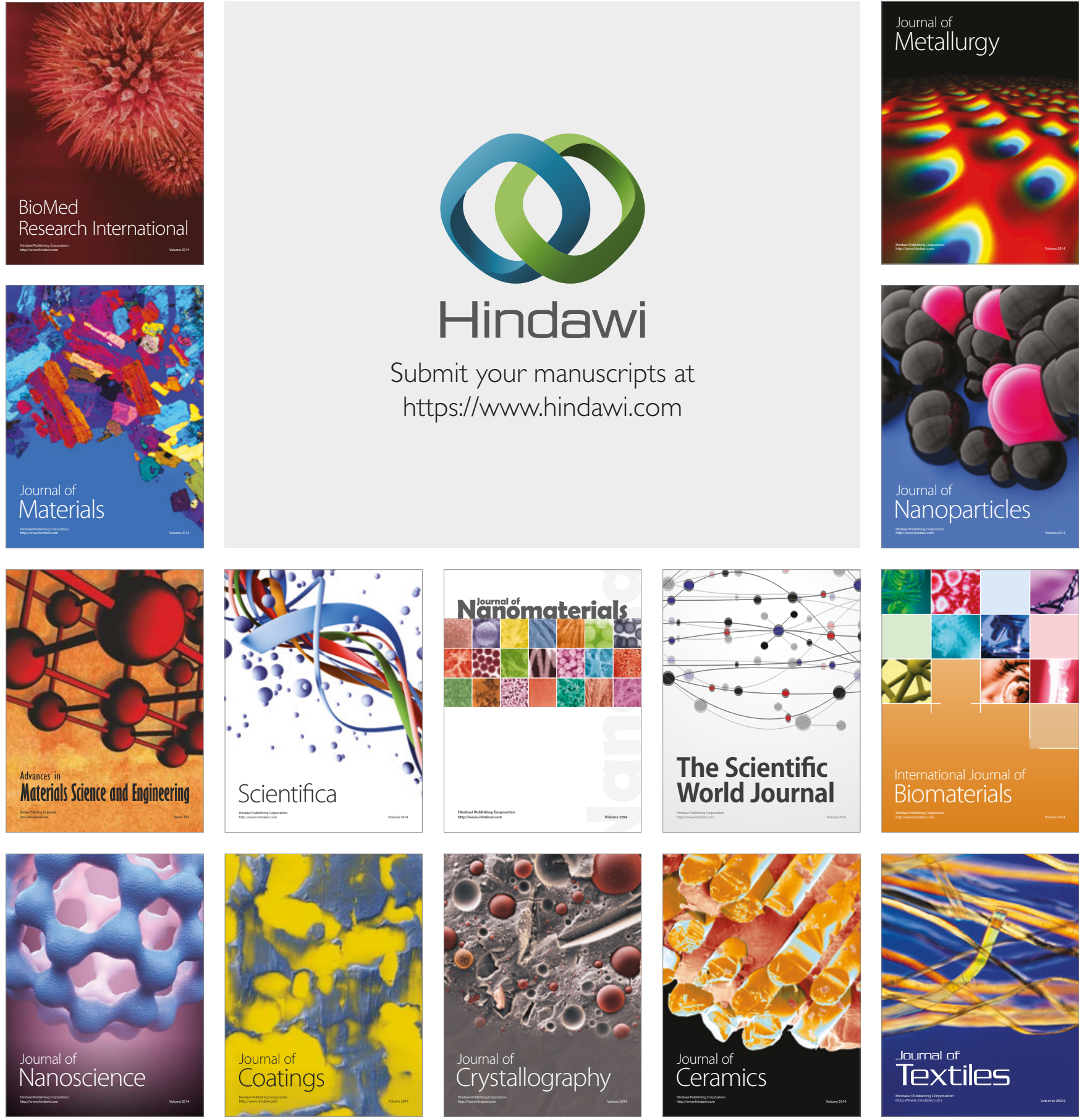

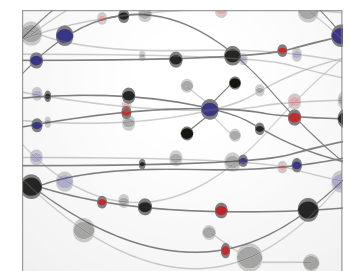

The Scientific World Journal
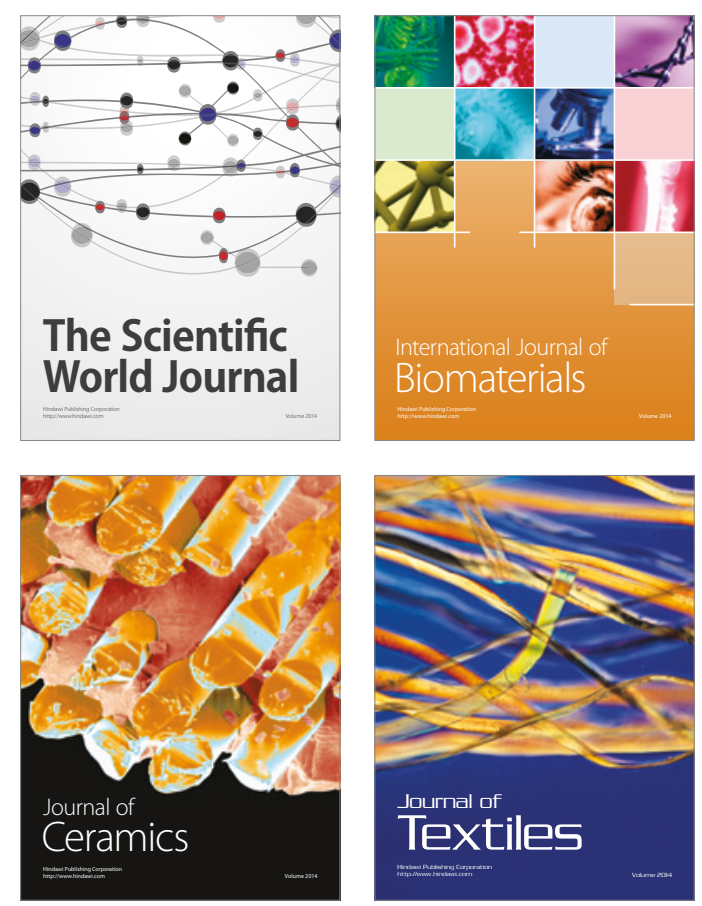